\title{
Single charge carrier type sensing with a parallel strip pseudo-Frisch-grid CdZnTe semiconductor radiation detector
}

\author{
D. S. McGregor, ${ }^{\text {a) }}$ Z. He, H. A. Seifert, and D. K. Wehe \\ Department of Nuclear Engineering and Radiological Sciences, University of Michigan, Ann Arbor, \\ Michigan 48109-2100
}

\author{
R. A. Rojeski \\ Etec Systems, Inc., 26460 Corporate Avenue, Hayward, California 94545
}

(Received 5 November 1997; accepted for publication 15 December 1997)

\begin{abstract}
An improvement in gamma-ray energy resolution is demonstrated for a room-temperature operated $\mathrm{CdZnTe}$ semiconductor detector that incorporates a parallel strip pseudo-Frisch-grid detector design. The device construction allows primarily for the measurement of electron charge carriers and not hole charge carriers. The detector is a three-terminal device that requires signal output to only one preamplifier, and its simplistic design offers an alternative method to single charge carrier type sensing to that of coplanar semiconductor gamma-ray detector designs. (C) 1998 American Institute of Physics. [S0003-6951(98)01807-5]
\end{abstract}

Wide band gap compound semiconducting materials offer great promise for use as room temperature operated radiation detectors. The most common semiconductor radiation detector design incorporates the use of a semiconducting block of material with metal contacts applied at opposite ends of the block. A voltage is applied across the block, which produces an internal electric field capable of drifting free charge carriers to the detector contacts. Gamma ray interactions occurring in the device excite electron-hole pairs which are separated by the applied electric field across the device bulk. Electrons are drifted towards the anode, and holes are drifted towards the cathode.

The induced charge produced by the moving free charge carriers can be measured by an external circuit. Shockley ${ }^{1}$ and Ramo $^{2}$ derived the dependence of the induced current and induced charge produced by point charges moving in an electric field, which was later shown to apply to semiconductor detectors as well. ${ }^{3-5}$ The Shockley-Ramo theorem states that the induced charge that appears at the terminals of a planar device from moving point charges is proportional to the distance displaced by the moving point charges, regardless of the presence of space charge. Hence, the change in induced charge $Q^{*}$ can be represented by

$$
\Delta Q^{*}=Q_{0} \frac{\left|\Delta x_{e}\right|+\left|\Delta x_{h}\right|}{W_{D}},
$$

where $Q_{0}$ is the initial charge excited by the interacting gamma ray, $W_{D}$ is the detector length, $\Delta x$ is the distance traveled by the electrons or holes, and the $e$ and $h$ subscripts refer to electrons or holes, respectively. The induced charge produced is a function of the complete motion of both electrons and holes, hence it is important in the operation of planar designed semiconductor radiation spectrometers that all electrons and holes be swept from the device. Charge carrier trapping creates a situation in which it is difficult to achieve full charge collection, hence energy resolution from

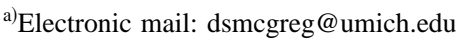

a semiconductor detector can be severely compromised. ${ }^{6,7}$ With trapping, the total induced charge from a singular gamma ray event in a planar semiconductor detector can be represented by

$$
\begin{aligned}
Q^{*}= & Q_{0}\left\{\rho_{e}\left(1-\exp \left[\left(x_{i}-W_{D}\right) / \rho_{e} W_{D}\right]\right)\right. \\
& \left.+\rho_{h}\left(1-\exp \left[-x_{i} / \rho_{h} W_{D}\right]\right)\right\},
\end{aligned}
$$

where $x_{i}$ represents the interaction location in the detector measured from the cathode, $\rho$ is the carrier extraction factor represented by

$$
\rho_{e, h}=\frac{\nu_{e, h} \tau_{e, h}^{*}}{W_{D}},
$$

where $\nu$ is the charge carrier velocity and $\tau^{*}$ is the carrier mean free drift time. From Eqs. (2) and (3), it becomes clear that the induced charge, $Q^{*}$, will be dependent on the location of the gamma ray interaction. Small values of $\rho$ for either holes or electrons will cause large deviations in $Q^{*}$ across the detector width. Generally, semiconductors have a much larger $\rho$ value for one carrier over the other. For $\mathrm{CdZnTe}$, the hole mobility $\left(120 \mathrm{~cm}^{2} / \mathrm{V} \mathrm{s}\right)$ and mean free drift time $\left(2 \times 10^{-7} \mathrm{~s}\right)$ are much less than the same values for electrons $\left(1350 \mathrm{~cm}^{2} / \mathrm{V} \mathrm{s}\right.$ and $10^{-6} \mathrm{~s}$, respectively). As a result, the value of $\rho_{e}$ can be 100 times greater than $\rho_{h}$. A $\mathrm{CdZnTe}$ detector that is sensitive to only electron motion and not hole motion would have an induced charge fluctuation dependent primarily on only the electron trapping losses.

A similar situation arises from the typically long pulse rise times observed with gas detectors. In a gas detector, gamma rays can ionize portions of the gas medium thereby producing electron-ion pairs. An electric field is applied across the gas volume to move the mobile charges. The average mobility of the ions is lower than the electrons, hence the observed pulse shape and induced charge become position dependent for typically used time constants. Frisch ${ }^{8}$ implemented a method to reduce position dependent charge collection effects in gas detectors by including a grid through the gas volume near the anode of the device (see Fig. 1). By 

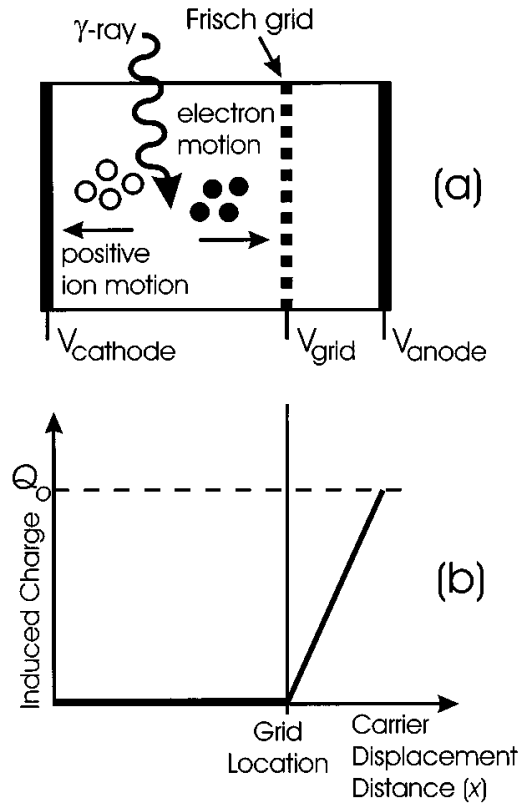

FIG. 1. The basic configuration for the Frisch grid gas detector. (a) The gas detector volume is separated into an interaction region and a measurement region. Electron charge carriers are drifted towards the anode by an applied bias. (b) The induced charge that appears at the anode is zero until the electrons pass through the grid. After passing through the grid, a charge is induced proportional to the distance traveled by the charge carriers between the grid and the anode.

biasing the electrodes properly, the electrons are drifted towards the anode through the grid. Positive ions are drifted in the opposite direction. According to the Shockley-Ramo theorem, the induced charge on the anode is primarily from electrons moving from the grid to the anode, hence, the gamma ray interaction position dependence on the pulse shape is greatly reduced.

A similar concept has been shown to work with coplanar semiconductor devices. ${ }^{9-13}$ Semiconductor coplanar detectors in the past have required the use of separate preamplifier circuits to subtract the signals induced on the separate sets of planar electrodes. ${ }^{9-13}$ However, recent developments on coplanar detectors allow for spectroscopic performance with only one preamplifier output. ${ }^{14}$ Here we implement a different approach to single charge carrier type sensing other than the coplanar grid device, in which we implement a parallel strip pseudo-Frisch-grid approach on a semiconductor device. As with a true Frisch grid, the electron charge carriers are transported from an interaction region, through a permeable grid region, and into an induced charge measuring region. The device requires only one output to a preamplifier and does not require a method of differentiating or subtracting pulses from parallel strips on a plane.

The prototype parallel Frisch grid semiconductor device has dimensions of $5 \mathrm{~mm} \times 2 \mathrm{~mm} \times 5 \mathrm{~mm} .{ }^{15}$ Electrodes were placed at opposite ends of two $2 \mathrm{~mm} \times 5 \mathrm{~mm}$ sides (see Fig. 2), thereby leaving $5 \mathrm{~mm}$ of bulk CdZnTe between the contacts. One such contact was operated as the anode and the other was operated as the cathode. Parallel conducting strips, each $0.5 \mathrm{~mm}$ wide, were then fabricated on the $5 \mathrm{~mm} \times 5 \mathrm{~mm}$ surfaces at a distance of $1 \mathrm{~mm}$ back from the anode contact (see Fig. 2). The region between the cathode and the parallel grid is the interaction region, the region under the grid is the

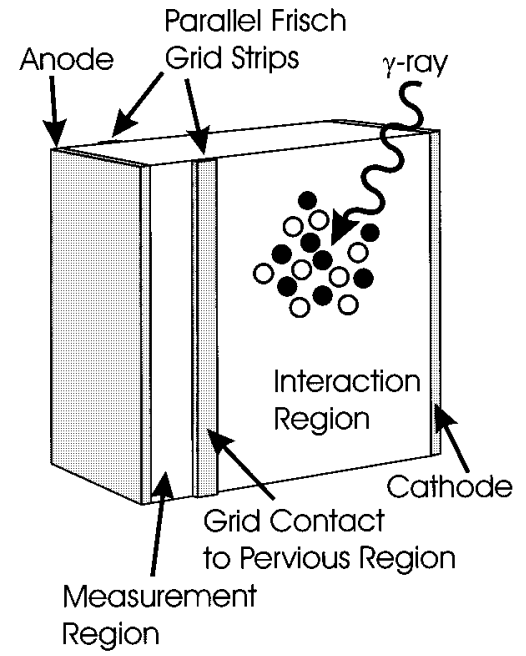

FIG. 2. Prototype semiconductor parallel strip pseudo-Frisch-grid design. The dimensions of the device are $5 \mathrm{~mm} \times 2 \mathrm{~mm} \times 5 \mathrm{~mm}$. Two of the $2 \mathrm{~mm} \times 5 \mathrm{~mm}$ ends have metal contacts covering them. Two parallel contact strips are fabricated on each of the $5 \mathrm{~mm} \times 5 \mathrm{~mm}$ faces opposite each other. The parallel strips are $0.5 \mathrm{~mm}$ wide and located $1 \mathrm{~mm}$ back from the anode. Charge carriers are excited in the interaction region and the electrons are drifted through the parallel Frisch grid to the measurement region by an applied electric field.

pervious region, and the region between the grid and the anode is the measurement region. Since the interaction region is much larger than the measurement region, the observed resolution is primarily due to gamma ray interactions that occur in the interaction region. Fluctuations of induced charge come primarily from electron trapping losses for gamma ray interactions that occur in the interaction region, and both electron and hole trapping losses for gamma ray interactions that occur in the measurement region.

The parallel strip pseudo-Frisch grid detector was tested in two modes, those being with the parallel grid off and the parallel grid on. In the "grid off" configuration, the anode was grounded through a $200 \mathrm{M} \Omega$ resistor, and the preamplifier circuit was ac coupled to the output lead between the anode and the bias resistor. The cathode was held at $-400 \mathrm{~V}$ through another $200 \mathrm{M} \Omega$ resistor, and the parallel grid was allowed to "float." In the "grid on" configuration, the anode was grounded through a $200 \mathrm{M} \Omega$ resistor with the preamplifier circuit ac coupled to the output (as before), the grid was held at $-80 \mathrm{~V}$ through a $200 \mathrm{M} \Omega$ resistor, and the cathode was again held at $-400 \mathrm{~V}$ through a $200 \mathrm{M} \Omega$ resistor. The biasing scheme divided the internal potential such that very little change in the internal electric field was developed from the grid off to grid on configurations.

Figure 3 shows the calculated weighting potential of the device, ${ }^{5,16}$ which clearly shows that most of the induced charge (95\% or more) will be produced as charge carriers move into the measurement region between the anode and parallel grid. Since electrons are drifted into the measurement region and holes are drifted away from the measurement region, the induced charge measured from the device output attached to the anode will be dependent primarily on the electron signal, and not the hole signal. Hence, the device will operate similar to a true Frisch grid, and it will also be sensitive primarily to the motion of electrons. The weighting potential shown in Fig. 3 indicates that charge carrier motion 


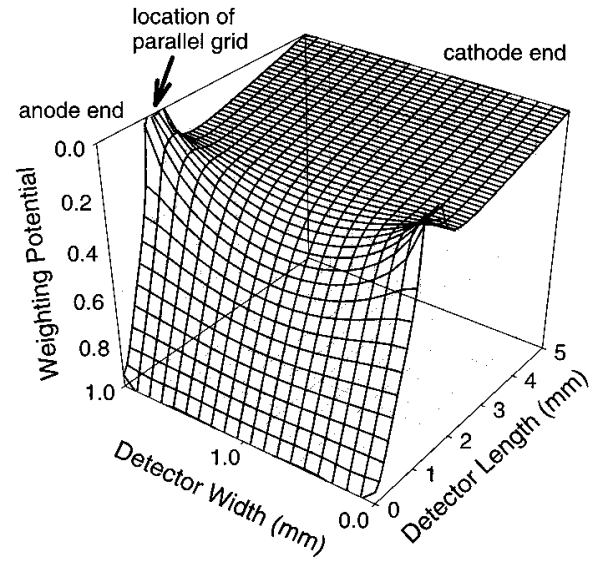

FIG. 3. The calculated weighting potential distribution across the parallel strip pseudo-Frisch-grid semiconductor detector with the grid activated. The weighting potential indicates that most of the induced charge is sensed as charge carriers move through the measurement region between the parallel grid and the anode. Since electrons are drifted to the measurement region from any location in the detector, most of the induced charge will be from electron motion.

in the interaction region will cause a small amount of charge to be induced on the anode, which is unlike a true Frish grid gas ionization chamber. Hence, we have used the term "pseudo-Frisch-grid" to describe the device.

Figure 4 shows $662 \mathrm{keV}$ gamma ray spectra taken from a calibrated ${ }^{137} \mathrm{Cs}$ source with the parallel Frisch grid off (floating) and on (at $-80 \mathrm{~V})$. With the grid off, the induced charge measured by the external circuit depends on the motion of both holes and electrons as they move between the anode and the cathode. From Fig. 4, it is clear that the resulting spectrum with the grid off suffers poor energy resolution from the position dependent charge collection (no discernable full energy peak). However, with the grid biased at

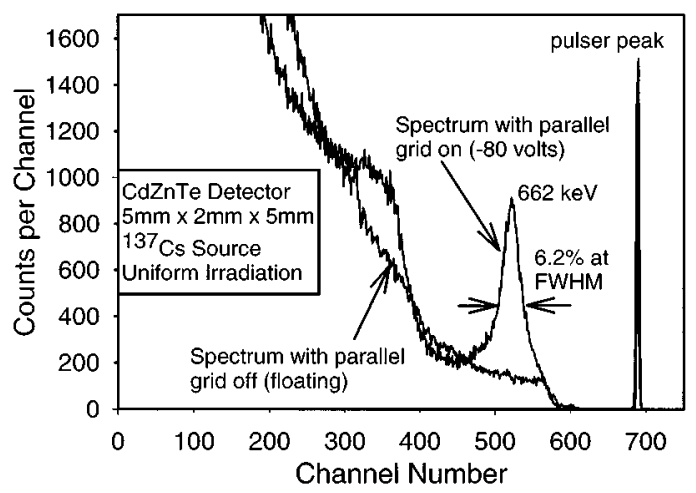

FIG. 4. Gamma ray spectra taken with $662 \mathrm{keV}$ gamma rays from a ${ }^{137} \mathrm{Cs}$ source. Shown are spectra taken with the parallel Frisch grid off (floating) and turned on (at $-80 \mathrm{~V}$ ). No full energy peak is apparent when the parallel grid is off, however a full energy peak with $6.2 \%$ energy resolution at FWHM becomes obvious when the grid is on. In both cases, the detector was uniformly irradiated. The operating temperature was $25{ }^{\circ} \mathrm{C}$ and the measurement time interval was $2.78 \mathrm{~h}(1000 \mathrm{~s})$.
$-80 \mathrm{~V}$, the induced charge measured by the external circuit depends only on the electron and hole movement within the measurement region. The energy resolution of the device was measured to be $6.2 \%$ at full width at half maximum (FWHM) while operating at a temperature of $25^{\circ} \mathrm{C}$. The measured induced charge is primarily from electrons that enter into the measurement region from the interaction region. Some pulses are measured for gamma ray interactions that occur within the measurement region, in which case both holes and electrons contribute to the signal. However, the distance that holes and electrons must travel for full charge collection in the measurement region is only $25 \%$ of the total detector length, hence the effects from position dependent charge collection can be minimized.

A 2-cm-thick lead attenuator was placed between the ${ }^{137} \mathrm{Cs}$ source and the area comprising both the pervious region and the measurement region. Gamma ray absorption events therefore occur primarily in the interaction region. Gamma ray spectra were taken with the Frisch grid off and on. A clearly resolved full energy peak (4.5\% energy resolution at FWHM) appeared only when the Frisch grid was activated, which demonstrates that the device is measuring electrons drifted into the measurement region from the interaction region, and not just the electron-hole pairs that were initially excited in the measurement region. Hence, the device performs similar to that of a true Frisch grid gas detector, but instead is a semiconductor radiation spectrometer counterpart.

Presently, the device operating bias voltages are limited by surface leakage currents. It is expected that gamma ray energy resolution will improve with better surface passivation techniques. Regardless, dramatic improvement in detector resolution is observed when the device is operated in Frisch grid mode, and the spectral measurements are achieved with only one output signal to the external circuit without the need for rise time rejection or correction techniques.

\footnotetext{
${ }^{1}$ W. Shockley, J. Appl. Phys. 9, 635 (1938).

${ }^{2}$ S. Ramo, Proc. IRE 27, 584 (1939).

${ }^{3}$ G. Cavelleri, G. Fabri, E. Gatti, and V. Svelto Nucl. Instrum. Methods 21, 177 (1963).

${ }^{4}$ M. Martini and G. Ottaviani Nucl. Instrum. Methods 67, 177 (1969).

${ }^{5}$ G. Cavelleri, G. Fabri, E. Gatti, and V. Svelto Nucl. Instrum. Methods 92, 137 (1971).

${ }^{6}$ R. B. Day, G. Dearnaley, and J. M. Palms IEEE Trans. Nucl. Sci. NS-14, 487 (1967).

${ }^{7}$ G. F. Knoll and D. S. McGregor, Proc. MRS 302, 3 (1993).

${ }^{8}$ O. Frisch, British Atomic Energy Report, BR-49, (1944).

${ }^{9}$ P. N. Luke, Appl. Phys. Lett. 65, 2884 (1994).

${ }^{10}$ P. N. Luke, IEEE Trans. Nucl. Sci. NS-42, 207 (1995).

${ }^{11}$ Z. He, Nucl. Instrum. Methods Phys. Res. A 365, 572 (1995).

${ }^{12}$ Z. He, G. F. Knoll, D. K. Wehe, R. Rojeski, C. H. Mastrangelo, M. Hammig, C. Barrett, and A. Uritani Nucl. Instrum. Methods Phys. Res. A 380, 228 (1996)

${ }^{13}$ Z. He, G. F. Knoll, D. K. Wehe, and J. Miyamoto Nucl. Instrum. Methods Phys. Res. A 388, 180 (1997).

${ }^{14}$ M. Amman and P. N. Luke Proc. SPIE 3115, 205 (1997).

${ }^{15}$ Custom fabricated as specified and obtained from eV Products, Saxonburg, PA.

${ }^{16}$ V. Radeka, Annu. Rev. Nucl. Part. Sci. 38, 217 (1988).
} 